\title{
Research on application of GPS-based wireless communication system in highway landslide
}

\author{
Zhiwen Xiong $^{*}$
}

\section{*Correspondence:}

hnlgdxxiongzhiwen@163. com

Department of Electrical Engineering, Guangxi

Technological College of Machinery and Electricity, Nanning 530007, People's

Republic of China

\begin{abstract}
Machine learning is a branch of the field of artificial intelligence. Deep learning is a complex machine learning algorithm that has unique advantages in image recognition, speech recognition, natural language processing, and industrial process control. Deep learning has It is widely used in the field of wireless communication. Prediction of geological disasters (such as landslides) is currently a difficult problem. Because landslides are difficult to detect in the early stage, this paper proposes a GPS-based wireless communication continuous detection system and applies it to landslide deformation monitoring to achieve early treatment and prevention. This article introduces the GPS multi-antenna detection system based on deep learning wireless communication, and introduces the time series analysis method and its application. The test results show that the GPS multi-antenna detection system of the wireless communication network has great advantages in response time, with high accuracy and small error. The horizontal accuracy is controlled at 0-2 $\mathrm{mm}$ and the vertical accuracy is about $1 \mathrm{~mm}$. The analysis method is simple and efficient, and can obtain good results for short-term deformation prediction.
\end{abstract}

Keywords: Deep learning, Wireless network communication, GPS detection system, Time series analysis, Data processing

\section{Introduction}

At present, frequent natural disasters around the world not only affect people's property safety, but also seriously affect people's lives and travel. If monitoring can be effectively carried out, it will be a good thing. Deep learning is a branch of machine learning. Its main principle is to use algorithms that contain complex structures or are composed of multiple nonlinear transformations to multiple processing layers to abstract data at a high level. In wireless networks, GPS technology is developing fastest. As a high-tech modern geodetic survey technology, GPS has become one of the most advanced deformation monitoring technologies and has been widely used due to its high-precision, fast, all-weather, and highly automated features. The text is mainly aimed at highway slope deformation. Research and analysis. party material in this article are included in the article's Creative Commons licence, unless indicated otherwise in a credit line to the material. If material is not included in the article's Creative Commons licence and your intended use is not permitted by statutory regulation or exceeds the permitted use, you will need to obtain permission directly from the copyright holder. To view a copy of this licence, visit http:// creativecommons.org/licenses/by/4.0/. 
Currently, there are two main modes of applying GPS to highway slope deformation detection. The first is the conventional monitoring mode of conventional GPS static relative positioning, and the second is the continuously operating station-type GPS monitoring system [1]. In the conventional monitoring mode, multiple GPS receivers are usually used to manually collect data point by point on a regular basis, and perform post-processing to obtain the deformation of each cycle, so as to predict the mid-to-long-term trend deformation of the slope. If the slope is already in an unstable state, it is necessary to use a continuously operating station GPS monitoring system to obtain the slope deformation status in real time [2]. However, the use of continuously operating stationtype GPS monitoring systems requires more investment in GPS hardware equipment, and the high price limits the application range of GPS technology. This article adopts the design scheme of GPS multi-antenna monitoring system, and connects multiple antenna arrays to the same receiver by attaching a GPS signal time-sharing connection switch without changing the existing GPS receiver structure. After the algorithm is processed, the deformation law of the deformable body can be obtained [3]. The GPS signal timesharing connection switch (called GPS multi-antenna switch) is used to switch between multiple antennas. The switching interval can be determined according to the state of the deformable body, ranging from a few seconds to a few hours. Since the price of the antenna is much lower than that of the receiver, the use of an antenna instead of a GPS receiver greatly reduces the cost of the GPS deformation monitoring system, making GPS technology a broad application prospect in deformation monitoring [4].

This article considers that landslide disasters mostly occur in dangerous or remote areas. In order to ensure personal safety and realize unattended working mode, the wireless communication network is used to transmit GPS monitoring data in real time and carry out GPS multi-antenna monitoring. To this end, we have done relevant research and developed a wireless communication network system that can quickly and accurately monitor slope deformation.

\section{Method}

\subsection{GPS}

\subsubsection{Brief description of GPS}

As a new generation of precise satellite positioning system, GPS system represents the cutting-edge technology and is the crystallization of the development of contemporary science and technology. Initially, GPS was originally developed for military applications, such as positioning and navigation of military vehicles, aircraft, and ships [5, 6]. Because of its ranging and timing functions, GPS can provide global users with high-precision, all-weather, and large-scale position and time information, which can well meet the military and civilian positioning and navigation needs. The composition of the GPS system is shown in Fig. 1.

As can be seen from Fig. 1, the GPS system is mainly composed of three parts: the space satellite part, the ground monitoring part, and the user receiving part [7].

(1) Space satellite part

The space satellite part is mainly composed of 24 satellites distributed in 6 elliptical orbital planes of the earth, including 21 working satellites and 3 standby satellites in orbit. These satellites are $17,700 \mathrm{~km}$ away from the earth and the satellite operating cycle 


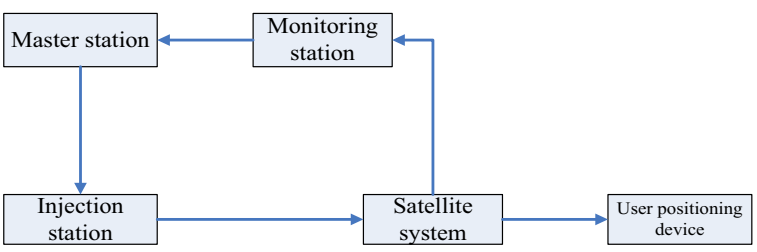

Fig. 1 GPS system composition diagram

is $1.58 \mathrm{~h}$. Four satellites are deployed on the plane, and the coverage angle is 55 degrees $[8,9]$. In actual use, the GPS system receiver can capture more than 4 satellites. At this time, in order to improve the positioning accuracy, the receiver divides the captured satellites into several groups according to the constellation distribution, with 4 satellites in each group. After calculation and analysis, the group with the smallest error is selected for positioning calculation.

(2) Ground monitoring part

The ground monitoring part is mainly composed of a main control station, 3 ground control stations and 5 global monitoring stations [10]. The main control station is located in Colorado, the United States mainland. The five monitoring stations distributed around the world under the direct control of the master control station are the data collection centers of the GPS system. Among them, the monitoring stations are equipped with receivers that can continuously measure visible satellite data and cesium clocks with precise time measurement. The main function of the monitoring station is to obtain satellite observation data including ionospheric and meteorological data and send it to the main control station. Then the main control station analyzes these data, calculates the clock parameters and satellite orbits, and sends the analysis results to three ground control stations.

(3) User receiving part

The user receiving part is mainly composed of GPS signal receiver [11]. The main function is to collect satellite signals. Through the collection and calculation of parameters such as the satellite orbit, the distance between the satellite and the receiver, the current position information of the user is obtained, including latitude and longitude, altitude, and speed of movement. GPS receiver is mainly composed of antenna and receiver. The receiver is powered by a DC power source inside and outside the machine. Generally, the power is supplied by an external power source and the battery is charged. After the power is turned off, the internal battery powers the memory to ensure data storage.

\subsubsection{GPS positioning principle}

GPS positioning methods are divided into two methods: absolute positioning and relative positioning. The former is used to determine the position of the moving carrier in the earth reference frame in real time. The positioning accuracy is within $100 \mathrm{~m}$. The latter uses multiple machines to determine the mutual relationship between measurement stations. After a certain period of observation, the data is processed by data postprocessing software, and its relative accuracy reaches nanometer level [12, 13].

(1) Absolute positioning principle 
The basic principle of the so-called absolute positioning is to use only one receiver to observe satellite signals and determine itself independently.

The position of the antenna phase center in the coordinate system is called absolute positioning because this position is the only absolute.

Positioning can be divided into dynamic absolute positioning and static absolute positioning $[13,14]$. The former is mainly used for flying because of its low positioning accuracy.

Navigation is required for machines, vehicles, and ships that require less precision. The latter can continuously measure the pseudo-range from the satellite to the observation station and improve the positioning accuracy through data processing, so it can be used for observation or navigation in some fine industries with high accuracy requirements [15].

GPS observation can get the position of the satellite and the distance from the satellite to the ranging point, and then use the satellite as the center and the distance as the radius to make a spherical surface. If three satellites are observed at the same time, we will get three spherical surfaces. It is the position of the measurement point that is required to be solved. Of course, in the actual measurement, due to the factor of the clock difference, the pseudo range measured by the receiver includes three coordinate component unknowns and one clock difference unknown, so if you want to solve these four unknowns, you must observe at least four satellites to establish The equations are used to settle the station coordinates corresponding to the user's receiver antenna. Let $\mathrm{P}$ be the pseudo-range observation, $\mathrm{R}$ be the true distance from the receiver to the satellite, $\mathrm{C}$ be the speed of light, and $\mathrm{T}$ be the difference in the reception clock, then the observation equation is:

$$
\rho=R+c+\tau=\sqrt{\left(x_{s}-x_{r}\right)^{2}+\left(y_{s}-y_{p}\right)^{2}+\left(z_{s}-z_{p}\right)^{2}}+c \times \tau
$$

(2) Relative positioning principle

The accuracy of absolute positioning is often inaccurate, which is mainly affected by factors such as satellite orbit errors, clock synchronization errors, and errors generated during propagation in the atmosphere. Although we can eliminate the errors caused by weakening some systems through methods such as mathematical modeling, its positioning accuracy can only reach meters, which is difficult to meet the needs of high precision [16]. Relative positioning is also called differential positioning. The basic principle is to use multiple receivers to observe GPS satellites simultaneously to determine the mutual relationship between the stations where each receiver is located in the earth coordinate system. Therefore, within a certain distance range, the orbit error of the satellite, the satellite clock error, the receiver clock error, and the refraction errors of the ionosphere and troposphere have a certain correlation with the impact on the observations. Use different combinations of these observations for relative positioning., The influence of the above errors can be eliminated or reduced, thereby improving the positioning accuracy.

In relative positioning, at least two GPS signal receivers are required, which are respectively set at the two ends of the baseline. One of the endpoints is a known coordinate point. The same set of GPS satellites are simultaneously observed, and the difference between the coordinate components between the two points and the baseline are 
measured. Length, the relative position of the baseline endpoint or the baseline vector is calculated, and the exact coordinates of the other point can also be calculated. Relative positioning can also be divided into static and dynamic positioning methods. The static relative positioning method is currently the most accurate of all GPS positioning methods, but the measurement time is relatively long, and generally takes one to three hours [17]. The dynamic relative positioning method is to press one receiver on a moving carrier and install the other receiver at a known point (reference station). The former is called a dynamic GPS signal receiver, while the latter is called a reference GPS signal receiver. These two receivers simultaneously observe a group of GPS satellites in sight, and the reference receiver provides differential correction numbers for the dynamic receiver, which is called GPS differential positioning data [18]. The dynamic receiver uses its own GPS observations and differential correction data from the reference receiver to accurately calculate the user's 3D coordinates.

\subsection{GPS multi-antenna detection system for wireless network communication}

\section{(1) GPS one-machine multi-antenna monitoring system}

The GPS one-machine multi-antenna monitoring system aims to give full play to the advantages of GPS measurement technology in automated real-time deformation monitoring and reduce the cost of purchasing GPS receivers. The design idea is: a GPS receiver is connected to multiple antennas, so that each Only GPS antennas are installed on the monitoring points, and no receivers are installed. Multiple monitoring points share a GPS receiver, which can greatly reduce the cost of the monitoring system without reducing the accuracy of conventional GPS measurements. Based on this idea, a GPS multi-antenna control switch can be designed so that one GPS receiver connects to multiple antennas, and these antennas work automatically in sequence by software control.

(2) Multi-antenna controller

The multi-antenna controller includes software and hardware, and is one of the core parts of a multi-antenna system. The hardware part is composed of multi-channel microwave switch and corresponding control circuit, a GPS receiver and corresponding processing chip; the off-state of several signal channels in the microwave switch is controlled by the switch control circuit in real time. The software part mainly realizes the functions of controlling the multi-channel working mode, setting the observation time of the measuring point, real-time communication with the GPS receiver and data transmission. The newly developed GPS multi-antenna controller, the field computer uses an embedded industrial control computer, and integrates the control circuit board and the dual-frequency GPSOEM board, and is equipped with an LCD liquid crystal display, which can intuitively monitor the situation of the multi-antenna data collection site.

The key technical problem to be solved in the hardware part of the GPS multi-antenna controller is the high isolation of the GPS signals of each channel in the microwave switch. The key technical problem to be solved in the software part is to realize real-time precise positioning, so that the positioning accuracy reaches $\mathrm{mm}$ level.

(3) Design of data transmission system

The data transmission from the GPS antenna to the multi-antenna controller can only be transmitted through a wired medium, so the coaxial cable or optical fiber can be used 
for data transmission. Coaxial cable is only suitable for short-distance data transmission; however, regardless of the distance of optical fiber, the quality and reliability of data transmission are guaranteed, but its cost is relatively high. The coaxial cable consists of a layer of mesh copper conductor and a copper conductor located on the central axis. Compared with the ordinary twisted pair, the coaxial cable has strong anti-interference ability and good shielding performance, and is often used for connection between devices. If a repeater (signal amplifier) is used, the length of the network connected by the coaxial cable can be increased up to several kilometers.

Data transmission from field data to the monitoring center. Since the monitoring site is generally located in a remote mountainous area, the field data received by the receiver adopts wireless transmission mode, GPRS and GSM are both good choices. Here we use the GPRS communication method. The specific method is to connect the GPS multiantenna controller through the RS-232 serial port on the controller and the GPRS terminal RS-232 serial port through a patch cord to transmit the original GPS data to GPRS terminal, and then continuously send to the monitoring center through the terminal wireless mode.

(4) Data processing system

The data processing system is responsible for the entire process of transmitting, storing, analyzing, calculating and displaying the original data of the receiver. First, the data of the multi-antenna receiver and the reference station are transmitted to the GPRS transmitter through the RS-232 serial port, and then the data is sent to the monitoring center through the wireless network long-distance transmission, and the monitoring center data processing software classifies and analyzes the multi-site data. Converted into location information. Through the process of comparing the position information of

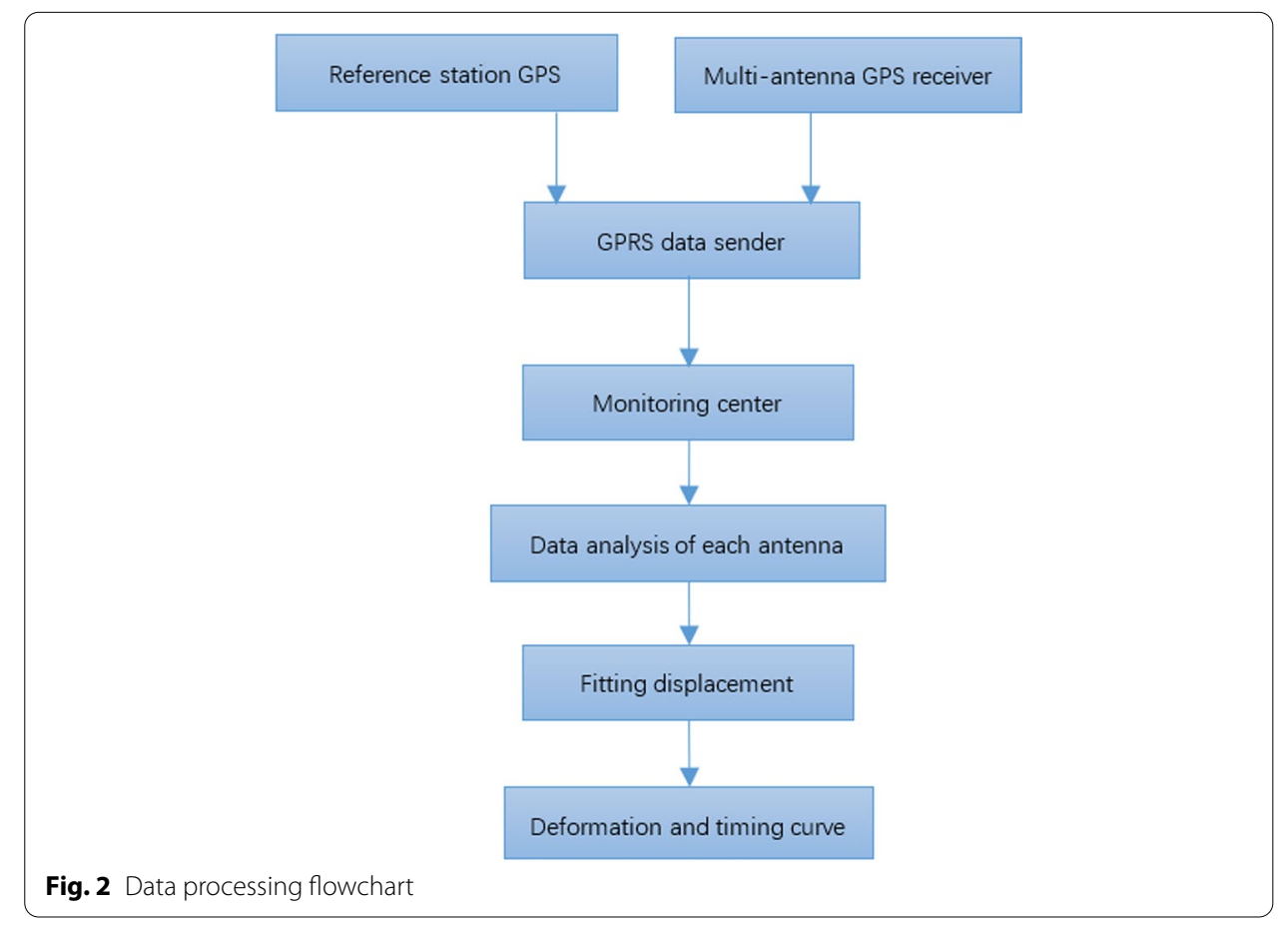


the reference station and the measuring point, and time sequence analysis, the predicted deformation trend curve can be generated, as shown in Fig. 2 [19].

\subsection{Inter sequence analysis}

For a long time, deformation analysis and processing methods have assumed that the observed data are statistically independent or uncorrelated, such as regression analysis methods. This kind of statistical method is a static data processing method, which cannot realize dynamic prediction of variables. However, whether it is observation data arranged in time series or observation data arranged in spatial order, there is more or less statistical autocorrelation between the data. With the development of modern science and technology and the improvement of computer application, various theories and methods have provided a wide range of research methods for deformation analysis and deformation prediction.

(1) Definition of stochastic process and time series

A stochastic process is a (family of) random variable that depends on a parameter. For example: the terminal voltage of an electronic component or device due to the random thermal disturbance of internal micro-particles is called thermal noise voltage, and its value at any given moment is a random variable; the temperature at each moment of the day is a random variable, which Sets constitute a random process. The definition of a stochastic process is: let $\mathrm{E}$ be a random test and $\mathrm{S}=(\mathrm{P})$ be its sample. If for each $\mathrm{e} \in \mathrm{s}$, there is always a real-valued function $\mathrm{X}(\mathrm{e}, \mathrm{t})$ Corresponding to this, the function of the parameter $\mathrm{t}$ of this family is called a random process, and each function in the family is called a sample function of the random process, and $\mathrm{T}$ is the variation range of the parameter $\mathrm{t}$, called a parameter set. Random processes can be divided into continuous random processes and discrete random processes according to whether they are continuous random variables or discrete random variables at any time. The specific value obtained by the random process in the test results is called the "implementation" of the random process, or the sample function, also called the sample observation. Time series are random sequences, that is, random sequences with discrete parameters.

(2) Time series modeling method

The key of time series analysis is to establish an appropriate mathematical model based on a reasonable analysis of observation results.

The general steps for modeling are:

(1) Preparation stage. The acquisition of initial data requires that the data can accurately and truly reflect the behavioral state of the modeling system. First, the data needs to be analyzed and tested, including the elimination of glitches and compensation data. The zero-mean test requires data preprocessing for sequences that do not meet the stability requirements. The processing methods mainly include differential processing or trend item extraction, and digital signal processing methods can process data flexibly.

(2) Preliminary determination of model structure and category. To determine the structure and category of the model, you need to choose a modeling method.

(3) After the structure of the model is determined, the appropriate method for selecting the model parameters should be estimated according to certain principles; then the model suitability test of the model is performed to determine the final appropriate model.

(3) The mathematical foundation of the model 
Auto-covariance function of random variables:

$$
D\left(X_{t}, X_{s}\right)=\operatorname{Cov}\left(X_{t}, X_{s}\right)=E\left\{\left[X_{t}-E\left(X_{t}\right)\right]\left[X_{s}-E\left(X_{s}\right)\right]\right\}
$$

Autocorrelation function and autocorrelation coefficient of random variables:

$$
R_{X}(t, s)=E\left(X_{t}, X_{s}\right)
$$

Initial estimation of model parameters:

$$
X_{t}=\phi_{1} X_{t-1}+\phi_{2} X_{t-2}+\cdots+\phi_{p} X_{t-p}+a_{t}
$$

Test of time series stationarity: According to the definition of stationary time series, the mean and variance of stationary time series are constant; the interval between selfcoordinates is related to the breakpoint of this interval. The relevant formula for the test of time series stationarity is as follows:

$$
\begin{aligned}
& \bar{X}_{i}=\frac{1}{M} \sum_{j=1}^{M} X_{i j} \\
& \hat{\sigma}_{i}^{2}=\frac{1}{M} \sum_{j=1}^{M}\left(X_{i}^{j}-\bar{X}_{i}\right)^{2} \\
& r_{\tau}(i)=\frac{1}{M} \sum_{j=1}^{M-\tau}\left(X_{i j}-\bar{X}_{i}\right)\left(X_{i, j+\tau}-\bar{X}_{i}\right) / \hat{\sigma}_{t}^{2}
\end{aligned}
$$

\section{(4) Model establishment}

According to the stationarity formula of the time series, the parsed data is firstly discretized, and then substituted into formula 5 to obtain the parameter mean value, the mean value is substituted into formula 6 to obtain the data variance, and finally substituted into formula 7 for stationarity analysis. After the above series of processing, Judging whether there is a trend of deformation according to the size of the difference.

\section{Discussion}

\subsection{Performance test and analysis of GPS detection system for wireless network communication}

(1) Response time analysis

In this article, the WGS84 coordinate system is used, and the origin is the center of mass of the earth. The $\mathrm{X}$ axis points to the intersection of the zero-degree meridian plane defined by the BIH and CTP equator, the $\mathrm{Y}$ axis and the $\mathrm{Z}$ axis, and the $\mathrm{X}$ axis constitutes a right-handed coordinate system. The response time of the GPS detection system used for wireless network communication is analyzed, and the result is shown in Fig. 3.

It can be seen from Fig. 2 that the response time of the GPS detection system for wireless network communication in this article is faster than the actual shortest time required. The response time of the system determines the performance of the system. 


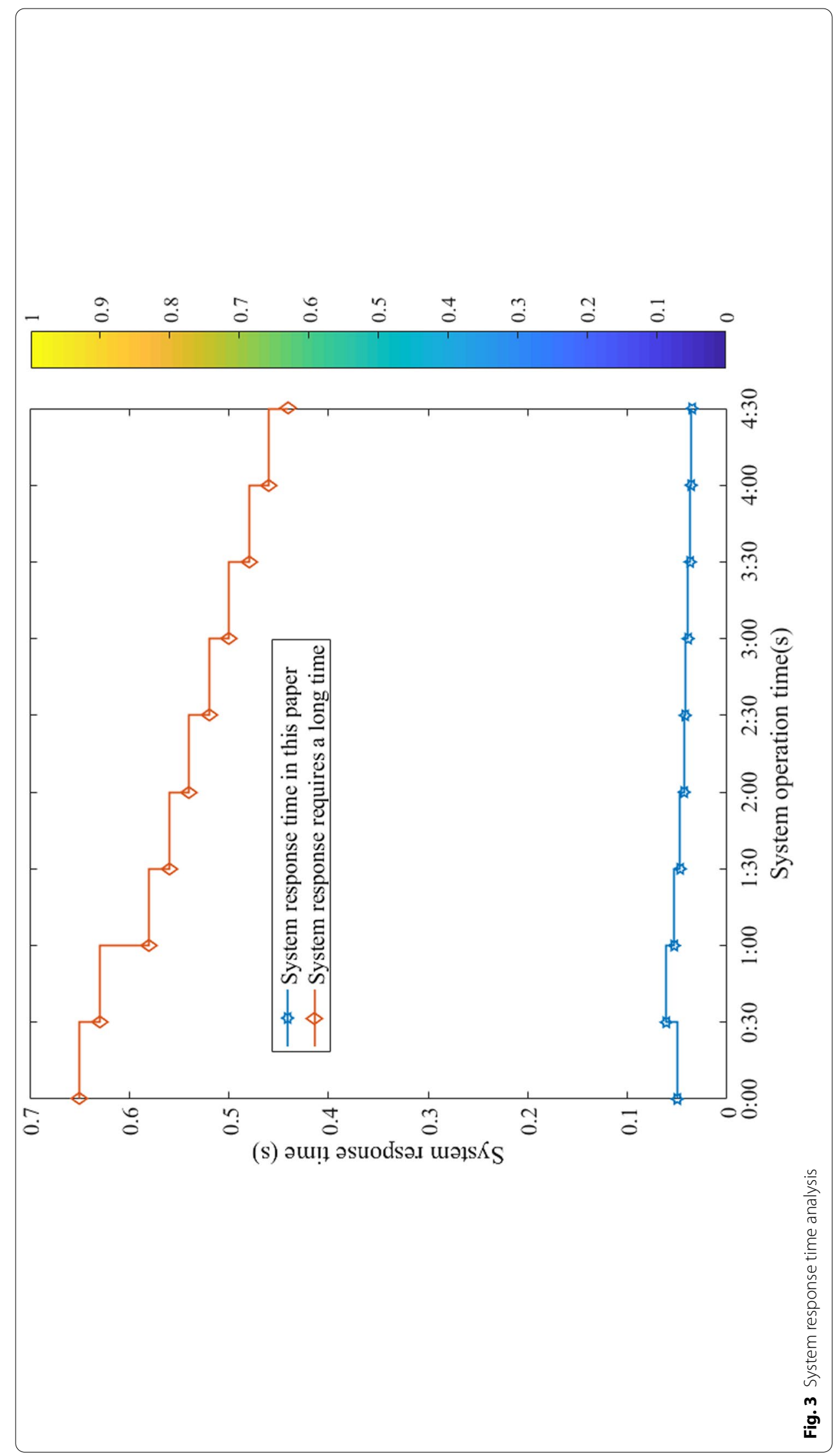


The GPS detection system for wireless network communication in this article has a great advantage in response time.

(2) Precision analysis

This paper uses GPSensor software to solve the data. At the beginning of the solution, the solution time is set to $15 \mathrm{~min}$. After the data is stable, the solution time is changed to $1 \mathrm{~min}$. After a certain observation, the GPS01 antenna direction X moves $10 \mathrm{~mm}$ to the north. Similarly, when the antenna switching controller switched to the channel corresponding to the GPS02 antenna, after a period of observation, GPS02 also moved $8 \mathrm{~mm}$ northward along X. After continuous testing, the results obtained are compared with the displacement to analyze the accuracy and sensitivity of the system. The results are shown in Fig. 4.

It can be seen from Fig. 3 that after analyzing the observation data of GPS01 and GPS02, the observation results show that the accuracy is: the horizontal accuracy is controlled at $0-2 \mathrm{~mm}$, and the vertical accuracy is about $1 \mathrm{~mm}$. It can be seen that the GPS detection system for wireless network communication in this article has a good advantage in accuracy.

\subsection{Analysis of highway slope deformation}

(1) Time series analysis

Take the GPS detection system of wireless network communication as an example to analyze and predict highway slope deformation, and compare the test results with the actual data results. First perform a time series analysis to determine the stability of the data. There are three ways to judge.

1) Use the unit root test method to check the stability of the data;

2) Observe the data line graph. If the line chart is irregular, or returns to a straight line infrequently, it means that the sequence is unstable;

3) Observe the sample autocorrelation function graph. If the sample autocorrelation function does not show an exponential decay trend, it indicates that the sequence is unstable. If the sequence is judged to be non-stationary, it can be segmented into a stationary sequence, but the difference should not be too large, otherwise the reversibility of the sequence will be destroyed, and the variance will increase, so the variance can be used to determine whether the difference is too large.

This article adopts the third method to establish and analyze the time series analysis model. The result is shown in Fig. 5. The values of the autocorrelation function and part of the autocorrelation function are shown in Table 1.

From the combination of Fig. 5 and Table 1, it can be seen that the original sequence diagram shows that the sequence has a clear trend. After two differences, a stable sequence was obtained, the model fitting accuracy was high, and the trend of the model fitting was basically the same as that of the original sequence. The stable sequence data indicated that the obtained slope did not present a risk of deformation.

(2) Analysis of application results

The GPS detection system of wireless network communication in this paper is used to predict the deformation of highway slope, and the results are shown in Fig. 6. 


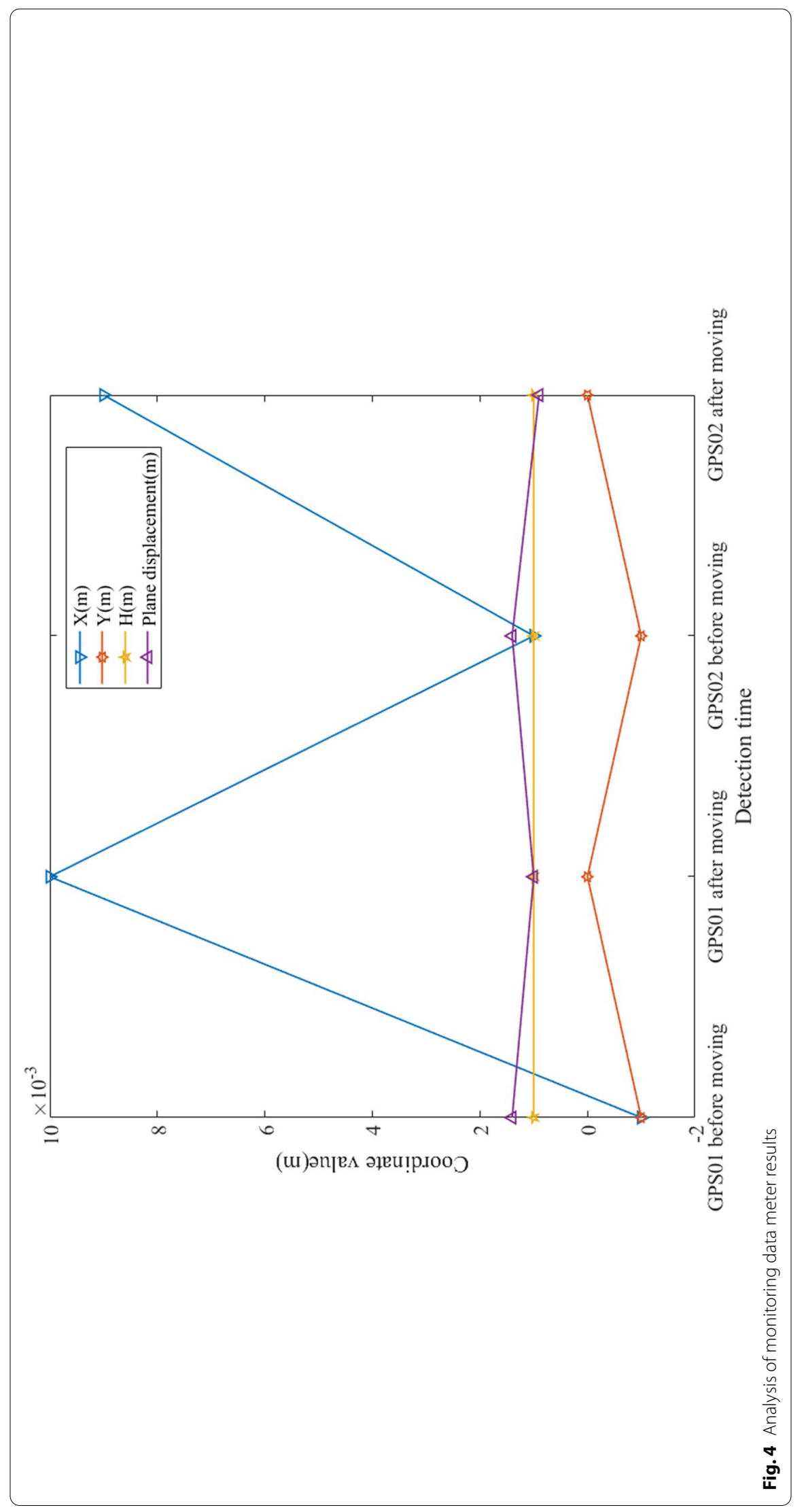




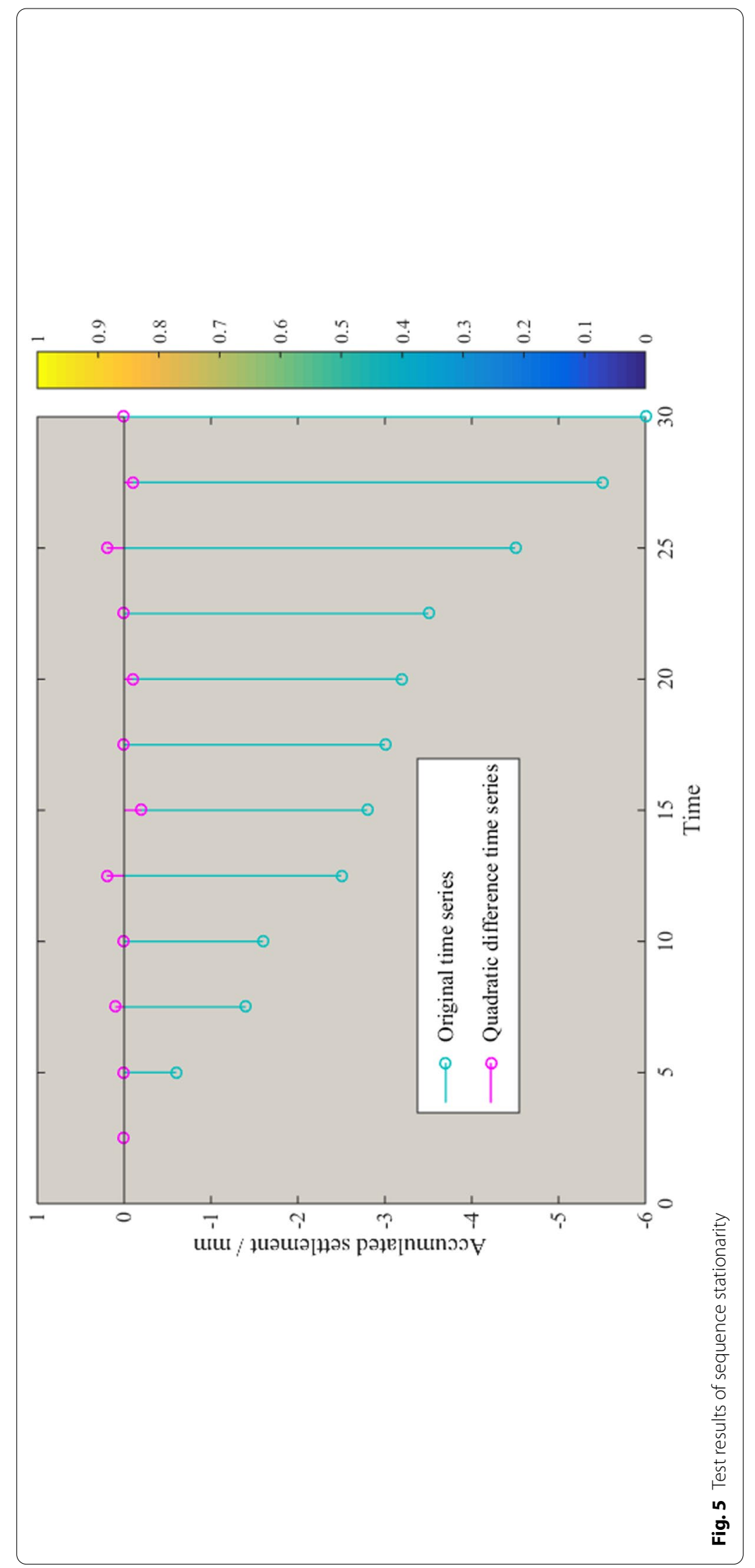


Table 1 Autocorrelation function and partial autocorrelation function values

\begin{tabular}{lllllll}
\hline$k$ & $\mathbf{0}$ & $\mathbf{1}$ & $\mathbf{2}$ & $\mathbf{3}$ & $\mathbf{4}$ & $\mathbf{5}$ \\
\hline Autocorrelation function & 1 & -0.405 & 0.007 & -0.023 & 0.080 & 0.057 \\
Partial correlation function & - & -0.536 & -0.301 & -0.226 & -0.213 & -0.77 \\
\hline
\end{tabular}

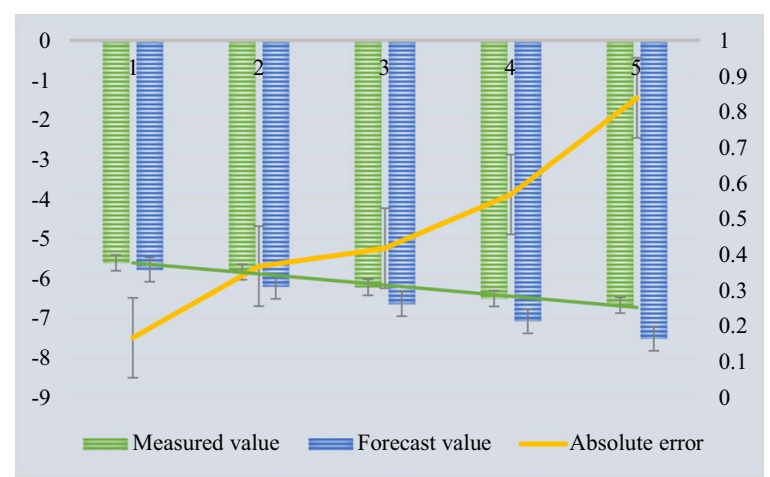

Fig. 6 Analysis of deformation and settlement detection and prediction results of highway slope

It can be known from Fig. 6 that the monitored deformation value of the highway slope is basically consistent with the predicted value, and the forecast accuracy decreases with the increase of the number of forecast steps. As an efficient method for modern dynamic data processing, intersequence has the advantages of easy modeling, simple calculation, fast prediction and high accuracy. Time series used in deformation monitoring can achieve higher fitting accuracy and prediction effect, especially for short-term prediction. Because the accuracy of the time series forecast decreases with the increase of the number of forecast steps, it is suitable for short-term forecasting; if medium- and longterm forecasting is required, the forecast results should be continuously revised using the measured data.

\section{Conclusions}

This text has carried on the systematic test to the GPS multi-antenna monitoring system of the wireless communication network. The research found that the wireless communication network GPS multi-antenna monitoring system in this paper has great advantages in system response time. In addition, in terms of accuracy, the GPS multi-antenna monitoring system of the wireless communication network in this article has higher detection accuracy and smaller errors. In addition, this paper conducted a case study. Although the reliability of the application examples was verified, the number of case samples was not comprehensive enough to explain the applicability of the system to complex terrain. Further testing and improvement are needed in the follow-up.

In addition, this paper also established a time series model for prediction, and verified the reliability of the model through experiments. Based on the verification results of the examples in this paper, considering the accuracy requirements and costs of landslide safety monitoring, the GPS multi-antenna monitoring system based on wireless communication network in this paper can automatically and continuously monitor landslide disasters, and can greatly reduce the overall The cost of the monitoring system is one of 
the ideal technologies for landslide and other geological disaster deformation monitoring, and can provide a certain paradigm role for the future geological disaster technology.

\section{Abbreviations}

WGS84: World geodetic system; GPS: Global positioning system; CTP: Agreement earth pole; ClO: Conventional international origin.

\section{Authors' contributions}

ZX carried out the research of GPS wireless communication continuous detection system, participated in time series analysis, established a time series model for prediction, and verified the reliability of the model through experiments and drafted a manuscript. The authors read and approved the final manuscript.

\section{Funding}

Funded by the Guangxi University Young and Middle-aged Teachers'Research Ability Improvement Project, project number: 2020KY32013; Funded by Scientific Research Project of Guangxi Mechanical and Electrical Vocational and Technical College, project number: 2019YKYZ002.

\section{Availability of data and materials}

Data sharing not applicable to this article as no datasets were generated or analysed during the current study.

\section{Declarations}

\section{Competing interests}

The authors declare that they have no competing interests in this section.

Received: 8 May 2021 Accepted: 2 August 2021

Published online: 10 August 2021

\section{References}

1. C. Alvarez, J. Díaz, J. Petit et al., High level communication functionalities for wireless sensor networks. Theor. Comput. Sci. 406(3), 240-247 (2016)

2. P. He, T. Fan, Distributed fault-tolerance consensus filtering in wireless sensor networks-Part I: communication failure. Int. J. Sens. Netw. 22(2), 127-142 (2016)

3. S. Randhawa, S. Jain, Energy-efficient load balancing scheme for two-tier communication in wireless sensor networks. J. Supercomput. 74(4), 1-31 (2017)

4. Y. Kwon, A. Kwasinski, A. Kwasinski, Coordinated energy management in resilient microgrids for wireless communication networks. IEEE J. Emerg. Sel. Top. Power Electron. 4(4), 1158-1173 (2016)

5. N. Wan, G.L. Kan, G.J. Wilson, Addressing location uncertainties in GPS-based activity monitoring: a methodological framework. Trans. GIS 21(4), 764 (2016)

6. S. Rungraengwajiake, P. Supnithi, S. Saito et al., lonospheric delay gradient monitoring for GBAS by GPS stations near Suvarnabhumi airport, Thailand. Radio Sci. 50(10), 1076-1085 (2016)

7. S. Alshorman, F.T. Jaber, F. Bensaali, A wireless oxygen saturation and heart rate monitoring and alarming system based on the Qatar early warning scoring system. J. Emerg. Med. Trauma Acute Care 2016(2), 155 (2016)

8. F. Yao, H. Wu, Y. Chen et al., Cluster-based collaborative spectrum sensing for energy harvesting cognitive wireless communication network. IEEE Access PP(99), 1-1 (2017)

9. P. Gupta, Correlating road network information and user mobility information for wireless communication network planning. Infect. Control Hosp. Epidemiol. 15(4), 280 (2017)

10. S. Mondal, S.K. Mohanty, S. Nandi, Energy efficient secure communication architecture for wireless sensor network: energy efficient secure communication architecture for wireless sensor network. Secur. Commun. Netw. 9(16), 3314-3323 (2016)

11. C. Liang, Q. Zhang, J. Ma et al., Research on neural network chaotic encryption algorithm in wireless network security communication. EURASIP J. Wirel. Commun. Netw. 2019(1), 151 (2019)

12. N. Sood et al., Integrating physics-based wireless propagation models and network protocol design for train communication systems. IEEE Trans. Antennas Propag. PP(99), 1-1 (2018)

13. S. Gopikrishnan, P. Priakanth, Retracted article: HSDA: hybrid communication for secure data aggregation in wireless sensor network. Wirel. Netw. 22(3), 1-18 (2017)

14. M. Singh, P.M. Khilar, A range free geometric technique for localization of wireless sensor network (WSN) based on controlled communication range. Wirel. Pers. Commun. 94(3), 1-27 (2017)

15. S. Bhowmik, C. Giri, A fuzzy communication model of sensor nodes in wireless sensor network. Int. J. Sens. Netw. 21(1), 1-15 (2016)

16. F. Afsana, Md. Asif-Ur-Rahman, M.R. Ahmed et al., An energy conserving routing scheme for wireless body sensor nanonetwork communication. IEEE Access PP(99), 1-1 (2018)

17. R. Costa, J. Lau, P. Portugal et al., Handling real-time communication in infrastructured IEEE 802.11 wireless networks: the RT-WiFi approach. J. Commun. Netw. 89(99), 1-15 (2019)

18. Y.-M. Ko, J.-H. Giil, H.-K. Song, An improved dual-hop transmission method for high reliability in wireless communication networks. Wirel. Pers. Commun. 97(9), 1-10 (2017) 
19. W. Li, Z. Qin, D. Xiaoli, D.W. Jiao, Y. Wentao, Z. Xianyun, GPS based on wireless communication network Antenna monitoring system and its application. J. Earth Sci. Environ. 31(3), 323-326 (2009)

\section{Publisher's Note}

Springer Nature remains neutral with regard to jurisdictional claims in published maps and institutional affiliations.

Submit your manuscript to a SpringerOpen ${ }^{\circ}$ journal and benefit from:

- Convenient online submission

- Rigorous peer review

- Open access: articles freely available online

- High visibility within the field

- Retaining the copyright to your article

Submit your next manuscript at $\boldsymbol{\nabla}$ springeropen.com 